\title{
Electrophysiological investigation of reward anticipation and outcome evaluation during slot machine play
}

\author{
SL Fryer ${ }^{\mathrm{a}, \mathrm{b}, *}$, BJ Roach ${ }^{\mathrm{b}}$, CB Holroyd ${ }^{\mathrm{c}}$, MP Paulus ${ }^{\mathrm{d}}$, K Sargent ${ }^{\mathrm{b}}$, A Boos ${ }^{\mathrm{b}}$, JM Ford ${ }^{\mathrm{a}, \mathrm{b}}$, \\ DH Mathalon ${ }^{\mathrm{a}, \mathrm{b}}$ \\ ${ }^{a}$ Department of Psychiatry and Behavioral Sciences, Weill Institute for Neurosciences, University of California, San Francisco, 401 Parnassus Avenue, San Francisco, CA \\ 94143, USA \\ ${ }^{\mathrm{b}}$ San Francisco VA Health Care System, San Francisco, CA, USA \\ ${ }^{c}$ Department of Experimental Psychology, Ghent University, Belgium \\ ${ }^{\mathrm{d}}$ Laureate Institute for Brain Research, Tulsa, OK, USA
}

\section{A R T I C L E I N F O}

\section{Keywords:}

Feedback related negativity (FRN)

Delta oscillations

Frontal medial theta oscillations

Near miss

Frustrative nonreward

\begin{abstract}
A B S T R A C T
Slot machines are a popular form of gambling, offering a tractable way to experimentally model reward processes. This study used a 3-reel slot paradigm to assess psychologically distinct phases of reward processing, reflecting anticipation, and early- and late-stage outcome processing. EEG measures of winning, nearly missing (a losing outcome revealed at the final, third reel), and "totally" missing (a losing outcome revealed earlier, at the second reel) were collected from healthy adults $(n=54)$. Condition effects were evaluated in: $i)$ event-related potential (ERP) components reflecting anticipatory attention (stimulus preceding negativity, SPN) and outcome processing (reward positivity, RewP and late-positive potential, LPP) and ii) total power and phase synchrony of theta and delta band oscillations. Behaviorally, trial initiation was fastest after a near miss outcome and slowest after a winning outcome. As expected, a significant SPN was observed for possible wins (AA) vs. total misses (AB), consistent with reward anticipation. Larger win (AAA) vs. near miss (AAB) amplitudes were observed for the RewP; LPP amplitudes were largest for wins (AAA), intermediate for near misses (AAB), and smallest for total misses (ABC), reflecting significant early (RewP) and late-stage (LPP) outcome processing effects. There was an effect of reel position on the RewP, with larger amplitude in the final reel (AAA-AAB) relative to the $2^{\text {nd }}$-reel locked difference waves (AA-AB). Across all outcomes, near misses elicited the largest and most phase-synchronized theta responses, while wins elicited larger and more phase-synchronized delta responses than total misses, with delta band measures not distinguishing between near misses and wins. . Phase locking measures contrasting win vs. near miss delta and theta synchronization, within time windows corresponding to ERP measurements, covaried with RewP, but not SPN or LPP, amplitude. Lastly, EEG measures showed differential relationships with age and selfreported consummatory pleasure. In the context of slot machine play, where reward anticipation and attainment place minimal demands on effort and skill, ERP and time-frequency methods capture distinct neurophysiological signatures of reward anticipation and outcome processing.
\end{abstract}

\section{Introduction}

Reward processing is a broad term that encompasses more specific sub-components that are increasingly appreciated on the basis of distinct underlying neurophysiological signatures. One major conceptual distinction parses reward-related functions into anticipatory processes related to reward responsiveness, motivation, and goal-oriented behaviors that have been referred to as "wanting," relative to consummatory processes related to reward attainment that have been referred to as "liking" (Berridge and Robinson, 2003). This distinction is reflected in dis- sociable neurocircuitry, with "wanting" responses more associated with distributed neuroanatomical circuitry mediated by dopaminergic neurotransmission and "liking" responses more associated with GABA, opioid, and endocannabinoid signaling (Berridge and Kringelbach, 2015).

Developing a more complete understanding of how reward-related neural measures relate to each other and underlie distinct psychological aspects of reward processing is important for characterizing reward responsivity in health and disease. Electroencephalography (EEG) can be used to parse in vivo human brain activity into constituent psychological processes with high temporal precision across phases of

\footnotetext{
* Corresponding author at: Department of Psychiatry and Behavioral Sciences, Weill Institute for Neurosciences, University of California, San Francisco, 401 Parnassus Avenue, San Francisco, CA 94143, USA.

E-mail address: Susanna.fryer@ucsf.edu (S. Fryer).
} 
reward processing. However, to date, many studies of reward-related EEG response have focused more narrowly on a single measure or time point (Glazer et al., 2018). In this study, we therefore combined traditional time-domain event-related potential (ERP) measures and timefrequency neuro-oscillatory measures to assess reward anticipation and early- and late stage outcome processing during slot machine play.

Electrophysiological measures of reward processing include the reward positivity (RewP), an ERP component that is larger to wins than losses (Gehring and Willoughby, 2002). The RewP has a frontocentral topography, peaking $\sim 250-300 \mathrm{~ms}$ after external feedback indicating reward outcome (Hauser et al., 2014, Holroyd and Umemoto, 2016, Proudfit, 2015, Walsh and Anderson, 2012), with simultaneously (Becker et al., 2014) and serially (Carlson et al., 2011) acquired fMRI demonstrating that feedback-locked RewP amplitudes covary with BOLD activations, including in ventral striatum, anterior cingulate cortex (ACC), and medial prefrontal cortex. The RewP had often been referred to as the feedback-related negativity (FRN), an evaluative component first elicited by feedback-locked analysis of errors in a time estimation task, (Miltner et al., 1997) which was subsequently shown to be larger to losses than wins in rewarded paradigms (Gehring and Willoughby, 2002). Because much of the ERP variability in this waveform appeared to derive from win events, with the loss-related negativity showing morphology and scalp topography similar to the N2 component of the N2-P3 complex elicited by shifts of attention to infrequent salient events (Holroyd et al., 2008) a reversal of the conditions historically subtracted to generate a difference wave (i.e., win-loss rather than loss-win) emphasized the relative positivity elicited by the win outcome (reviewed by (Proudfit, 2015)). The RewP is thought to reflect receipt of midbrain dopaminergic reward prediction error signaling by the ACC, tracking magnitude and valence of expected vs. attained rewards and therefore encoding outcomes as better or worse than expected (Holroyd and Coles, 2002). As such, the RewP may subserve reinforcement learning (Holroyd and Umemoto, 2016) as an electrophysiological index of prediction error-based reward valuation (Hauser et al., 2014, Walsh and Anderson, 2012). Although ERP studies of reward processing have largely focused on the RewP to date, interest is gaining in expanding assessments to include later components that reflect affective responses to reward outcomes (Glazer et al., 2018), such as the late positive potential (LPP) (Glazer et al., 2018, Cockburn and Holroyd, 2018), as well as components preceding the outcome that reflect reward anticipation, such as the stimulus-preceding negativity (SPN), a measure of anticipatory attention (Glazer et al., 2018, Brunia et al., 2011, Brunia and van Boxtel, 2001). And more granular efforts to parse anticipatory from consummatory sub-stages of reward processing with ERPs (Novak and Foti, 2015) have revealed ERPs that selectively covary with traits relevant for reward processing (e.g., (Novak et al., 2016)).

In addition to ERPs, time-frequency measures of neuro-oscillatory power and phase synchrony have been useful in characterizing component processes related to reward responsivity. Frontomedial theta oscillations, more broadly associated with cognitive control and error processing (Holroyd and Umemoto, 2016, Cavanagh and Frank, 2014), are responsive to anticipatory and evaluative phases of reward processing, particularly in association with losses and loss-related learning (Bernat et al., 2015, Bernat et al., 2011, Cavanagh et al., 2010, Cohen et al., 2007, Gruber et al., 2013, Marco-Pallares et al., 2008). In contrast, posterior delta oscillations are maximally responsive to winning outcomes (Bernat et al., 2015, Bernat et al., 2011), possibly in connection with positive prediction error formation and subsequent behavioral adjustments (Cavanagh, 2015). More specifically, prior work examining frequency-specific neuro-oscillatory EEG activity within the RewP time window has demonstrated that delta and theta evoked power are associated with ERPs to wins and losses, respectively (Watts et al., 2018, Foti et al., 2015, Nelson et al., 2011) providing convergent evidence that neural mechanisms underlying feedback processing differ by outcome (but see (Christie and Tata, 2009)). The potential practical value of combining ERP and time-frequency measures is illustrated by a recent clinical study of reward processing in which combining time frequency-based measures of delta oscillations with the RewP enhanced the sensitivity and positive predictive value of depression risk models (Nelson et al., 2018).

Many previous neuroimaging studies examining reward processing have used paradigms that deliver rewards based on the participant's behavioral performance and/or decision making such that rewards earned depend on participant action selection and motor responding (e.g. (Knutson et al., 2000)). Studies based on these and similar tasks make valuable contributions in modeling reward attainment under conditions requiring these higher-order processes. For example, metaanalysis indicates the RewP is positively modulated by the extent of personal control over outcome (Sambrook and Goslin, 2015). Nevertheless, performance-based reward tasks pose an inherent challenge in isolating more basic reward processes from other features such as the individual's motivation, cognitive effort, and performance skill. Modeling more basic reward features is not only relevant to understanding healthy reward functions, but is necessary to develop a more precise understanding of reward system dysfunctions in neuropsychiatric disorders, or other populations with co-occurring cognitive and motivational impairments (Barch et al., 2016, Whitton et al., 2015, Nusslock and Alloy, 2017).

It has previously been demonstrated that a RewP can be elicited by slot machine-style tasks in which reward outcomes do not depend on decision-making or motor responding (Donkers et al., 2005, Donkers and Van Boxtel, 2005), offering a compelling, real-word analog of elemental reward processes. Slot machines are a common and highly reinforcing form of gambling that typically feature near miss outcomes, in addition to wins and complete losses. Near misses are outcomes that have closer proximity to a win than a "total" loss, but have the same economic value as losses; they are typically experienced as more aversive but also more motivating than wins, in that they increase play persistence (Barton et al., 2017, Parke and Griffiths, 2006, Reid, 1996). Here, we used a slot machine paradigm to evaluate basic aspects of reward anticipation and outcome processing, including responses to near misses. The goals of the present study were to use a comprehensive set of EEG measures to parse psychologically distinct phases of reward processing while minimizing performance demands that complicate interpretation of many prior studies of reward processing. We further assessed whether electrophysiological measures could identify response differences between near and total misses, suggesting that those measures reflect aspects of reward processing beyond strict economic valuation of outcomes. Accordingly, we combined analysis of ERPs reflecting anticipatory attention allocation (SPN) and early (RewP) and late (LPP) stages of reward outcome evaluation with time-frequency analysis (TFA) of theta and delta oscillatory total power and phase synchronization during time windows corresponding to the ERP measurements. After first characterizing the paradigm with time-domain ERP components, ERPs were then related to event-related magnitude changes (total power) and event-related phase synchronization and also to individual differences in age and anticipatory and consummatory reward sensitivity. In addition to establishing expected significant condition effects for the SPN, RewP, and LPP, we hypothesized that theta and delta measures would explain significant variance in the time-domain RewP, and that this broad measurement set would explain variance in participant age and trait reward sensitivity.

\section{Methods}

\subsection{Study participants}

Data were collected from 54 (42 male) healthy adults between the ages 19 and $61(33.72 \pm 14.4)$, recruited from the community through online advertisements, flyers, and word-of-mouth. The Structured Clinical Interview for DSM-IV (SCID-IV-TR) (First et al., 2002) ruled out current or past Axis I psychiatric disorders. Recent substance use for 


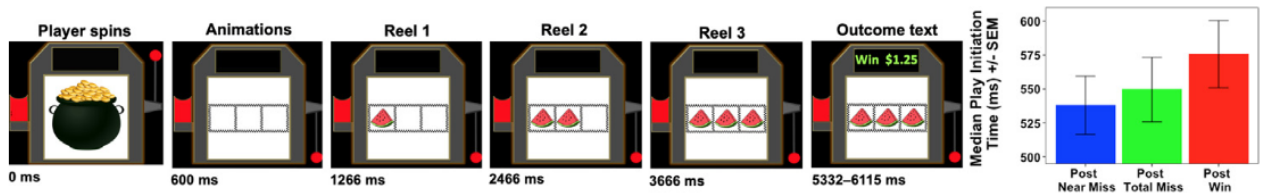

Fig. 1. Top: Slot machine task timing diagram: evolution of a single trial. Bottom: Bar graph of player initiation times by trial type. common testable drugs of abuse (e.g., cannabinoids, opiates, cocaine, amphetamines) was ruled out through urinalysis on assessment days. English fluency, and 18-65 years of age were required. History of head injury, neurological illness, or other major medical illnesses that affect the central nervous system were exclusionary criteria. All participants provided written informed consent under procedures approved by the Institutional Review Board at University of California, San Francisco (UCSF).

\subsection{Slot machine task}

Participants completed 288 trials of a slot machine reward processing task developed for this study and informed by prior work (Donkers et al., 2005, Donkers and Van Boxtel, 2005, Clark et al., 2009, Dymond et al., 2014, Habib and Dixon, 2010). To build expectancy, the display consisted of 3 sequentially populated slot reel positions (stimulus onset asynchrony; $\mathrm{SOA}=1.2 \mathrm{sec}$ ). Each reel was initially blank until populated with one of 12 possible fruit symbols, culled from a royalty free-clip art library, https://openclipart.org. Fruit symbols were distributed equally among possible outcomes, such that individual fruit symbols carried no predictive information about likelihood of winning. Total trial time after the participant initiated a given play was $6115 \mathrm{~ms}$ (see Fig. 1 for detailed trial timing).

To increase face and ecological validity, specific task design features were included to mimic structural characteristics common to real-word slot machines, including sound effects (audible coin drop) and visualizations (coin insertion, lever pull, visual flicker during play outcome) (Parke and Griffiths, 2006, Griffiths, 1993). In order to promote a sense of agency and control which positively modulates the signals being studied (Sambrook and Goslin, 2015, Clark et al., 2009), trials were initiated via participant button press, after which timing of the slot reels was automated so that reward outcomes did not depend on participant decisionmaking or motor response preparation or execution. More specifically, the participant's button press triggered an audible animated coin drop and lever press, followed by reel positions being populated sequentially, whereby reels 1 (R1), R2, and R3 were each populated with a single fruit symbol. After R3 populated, the outcome (reward evaluation) phase began and a visual checkerboard border flickered for $1000 \mathrm{~ms}$ followed by outcome text that depicted either "WIN \$1.25" or "LOSE," depending on the trial type. There were 3 trial types, presented in a pseudorandom sequence: frequent losses evident at R2 (total miss probability $=.50$ ), infrequent wins evident at R3 (win probability $=.25$ ), and infrequent near misses evident at R3 (near miss probability $=.25$ ). Wins (AAA) occurred when 3 identical fruit symbols were populated in the 3 slot reels; Near Misses (AAB) occurred when the first and second reel symbols matched but the R3 symbol did not match (AAB); Total Misses (ABC) occurred when the R2 symbol did not match the R1 symbol, indicating a loss at R2, with the R3 outcome providing no additional information about the loss. Near Miss and Total Miss trials were $\$ 0$ payouts, whereas each Win trial yielded a $\$ 1.25$ payout. To ensure that participants would feel that the opportunity for reward was valid, they played for a monetary bonus that reflected a portion of their slot machine winnings above their regular compensation for study participation.

\subsection{Hedonic trait sensitivity}

The Temporal Experience of Pleasure Scale (TEPS) is self-report scale used to measure anticipatory and consummatory experiences of plea- sure on a scale from 1 ("very false for me") to 6 ("very true for me") (Gard et al., 2006). Higher scores indicate higher levels of experiential pleasure (total score range $0-28$ ). The anticipatory subscale (10 items; alpha $=.76)$ primarily reflects receptiveness to rewards (e.g., "wanting", and the consummatory subscale $(8$ items; alpha $=.64$ ) reflects hedonic response (e.g. "liking") and inversely relates to anhedonia. Test-retest reliability over $\sim 6$ weeks for the subscales was .80 and .75 , respectively (Gard et al., 2006).

\subsection{EEG data acquisition and processing}

EEG data were recorded from 64 channels using a BioSemi ActiveTwo system (www.biosemi.com). Electrodes placed at the outer canthi of both eyes, and above and below the right eye, were used to record vertical and horizontal electro-oculogram (EOG) data. EEG data were continuously digitized at $1024 \mathrm{~Hz}$ and referenced offline to averaged mastoid electrodes before applying a $0.1 \mathrm{~Hz}$ high-pass filter using ERPlab (Lopez-Calderon and Luck, 2014). Data were next subjected to Fully Automated Statistical Thresholding for EEG artifact Rejection (FASTER) (Nolan et al., 2010). The method employs multiple descriptive measures to search for statistical outliers ( $> \pm 3$ SD from mean): (1) outlier channels were identified and replaced with interpolated values in continuous data, (2) outlier epochs were removed from participants' single trial set, (3) spatial independent components analysis was applied to remaining trials, outlier components were identified using the ADJUST procedure (Mognon et al., 2011), data were back-projected without these components, and (4) outlier channels were removed and interpolated within an epoch. The original FASTER processing approach was modified between steps 2 and 3 to include canonical correlation analysis (CCA). CCA was used as a blind source separation technique to remove broadband or electromyographic noise from single trial EEG data, generating denoised EEG epochs. This approach is identical to the CCA method described in our prior reports (Kort et al., 2017, Mathalon et al., 2018). Of the 288 trials presented, $278.19+/-10.93$ were acceptable after artifact rejection and were subjected to analysis; condition-specific breakdown of trials subjected to analysis were: $69.67+/-3.14$ wins, $69.78+/-3.02$ near misses, and $138.74+/-5.50$ total misses.

ERP Measurement Reel 3. Epochs were time-locked to the onset of R3 and baseline corrected using either the -100 to $0 \mathrm{~ms}$ baseline preceding R3 for the RewP and LPP, which were measured post-R3, or the -2400 to $-2300 \mathrm{~ms}$ period preceding R3 (i.e., -100 to $0 \mathrm{~ms}$ preceding R2) for the SPN, which was measured pre-R3. ERP averages were generated using a trimmed means approach, excluding the top and bottom $10 \%$ of single trial values at every data sample in the epoch before averaging to produce a more robust mean estimation (Leonowicz et al., 2005). The SPN was measured as the average area in the window from -100 to 0ms prior to the R3 outcome (Brunia et al., 2011, Brunia and van Boxtel, 2001, Donkers et al., 2005, Donkers and Van Boxtel, 2005), with a separate ERP average of the AA trials (collapsed across AAA and AAB trials) and the $\mathrm{AB}$ trials (i.e., the $\mathrm{ABC}$ trials). The RewP was measured as the average voltage between $228-344 \mathrm{~ms}$ post $\mathrm{R} 3$ onset in the AAA-AAB difference wave, based on a time window established by meta-analysis of the RewP across 54 studies (Sambrook and Goslin, 2015). The LPP to $\mathrm{AAA}, \mathrm{AAB}$, and $\mathrm{ABC}$ trials was calculated as the average voltage between 600-800ms (Hajcak and Olvet, 2008) after R3.

Reel 2. In addition to the R3-locked RewP, a RewP was measured as the average voltage between $228-334 \mathrm{~ms}$ post $\mathrm{R} 2$ in the AA-AB difference wave in order to compare the brain's response to outcomes revealed 
at R3 (i.e., win $\mathrm{AAA}$ vs. near miss $\mathrm{AAB}$ ) with outcomes revealed at R2 (i.e., possible win AA vs. total miss AB). LPP amplitudes were not similarly measured in response to R2 because the LPP time window overlaps with the SPN.

For statistical analyses, measurements were based on representative electrodes commonly used in the literature $(\mathrm{FCz}$ for RewP (Holroyd et al., 2008, Cockburn and Holroyd, 2018, Donkers et al., 2005, Donkers and Van Boxtel, 2005), Cz for SPN (Donkers et al., 2005, Donkers and Van Boxtel, 2005), and Pz for the LPP (Glazer et al., 2018)).

Time-Frequency Analysis (TFA): Time frequency analysis of single trial EEG data was done with Morlet wavelet decomposition using FieldTrip software (Oostenveld et al., 2011) in MATLAB. Specifically, we used a Morlet wavelet with a Gaussian shape defined by a ratio $\left(\sigma_{\mathrm{f}}=\mathrm{f} / \mathrm{C}\right)$ and $6 \sigma_{\mathrm{t}}$ duration (f is the center frequency and $\left.\sigma_{\mathrm{t}}=1 /\left(2 \pi \sigma_{\mathrm{f}}\right)\right)$. In this approach, as the frequency (f) increases, the spectral bandwidth $\left(6 \sigma_{\mathrm{f}}\right)$ increases. Center frequencies were set to minimize spectral overlap for two frequency bins: delta $=3 \mathrm{~Hz}$ (range: $2.5-3.5 \mathrm{~Hz}$ ) and theta $=5$ $\mathrm{Hz}$ (range: 4.2-5.8 Hz). Inter-trial coherence (ITC) of phase was calculated as 1-minus the circular phase angle variance (Tallon-Baudry et al., 1997). ITC provides a measure of the phase consistency of frequency specific oscillations with respect to stimulus onset across trials on a millisecond basis. Total power was calculated by averaging the squared single trial magnitude values in each frequency bin on a millisecond basis. The average power values were $10 \log _{10}$ transformed and then baseline corrected by subtracting the mean of the pre-R2 stimulus baseline $(-250$ to $-150 \mathrm{~ms})$ from each time point separately for every frequency. The resulting values describe change in total power relative to baseline in decibels (dB). For each participant, time-frequency measures (power, ITC) were extracted from delta $(3 \mathrm{~Hz})$ and theta $(5 \mathrm{~Hz})$ bands in the same time windows corresponding to the ERP components of interest (i.e., SPN, RewP, LPP) and subjected to analyses as described below.

\subsection{Data analysis plan}

Task behavior: For behavioral data, repeated measures analysis of variance (ANOVA) was used to compare median response time to initiate trials after wins (AAA), near misses (AAB) and total misses (ABC). Bonferroni correction was applied to control familywise type 1 error for pairwise follow-up comparisons across the three conditions $(\mathrm{p}<.0167)$.

ERP and TFA Condition effects: ERP amplitude measures for the SPN (AA vs. $\mathrm{AB}$ ) and RewP (AAA vs. AAB) were assessed with t-tests, while the LPP was assessed with a repeated measures ANOVA (AAA, $A A B, A B C)$. Unlike the ERP waveforms, which exhibited expected complex morphologies that greatly differed between R2 and R3, the timefrequency decomposition produced simplified waveforms that enabled examination of single condition effects to assess the impact of the three reward outcomes (win AAA at R3, near miss $\mathrm{AAB}$ at R3, total miss $\mathrm{AB}$ at R2) on underlying signals. Therefore, we conducted 4 separate condition TFA ANOVAs (each with three levels of outcome) for theta and delta power and ITC measures. Total miss (AB) trials were downsampled to avoid confounding trial number in these comparisons. Multiple comparisons correction strategy: Our approach to controlling for Type 1 error for primary ERP and TFA condition effect analyses was to apply Bonferroni-correction across the entire family of 7 EEG comparisons (i) SPN, ii) RewP, iii) repeated measures for LPP (wins, near misses, total misses), and iv-vii) repeated measures theta and delta power and ITC (wins, near misses, total misses). Follow-up LPP and TFA analyses were also Bonferroni-corrected to account for three repeated measures within these comparisons (all follow-up p values were held to the Bonferronicorrected alpha, $\mathrm{p}<.0167)$.

\subsubsection{ERP and TFA Relationships}

The relationships between ERP and TFA measures were assessed using separate multiple regression models predicting four ERP measures (SPN, RewP, LPP_wins, LPP_near misses) with the four delta and theta
Table 1

Participant demographic characteristics and play initiation times.

\begin{tabular}{ll}
\hline \multicolumn{2}{l}{ Demographic Data } \\
\hline $\mathbf{n}$ & 54 \\
\hline Mean Age +/- S.D. (years) & $33.72+/-14.4$ \\
& (range: $19-61)$ \\
Sex (\% male) & $77.7 \%$ \\
Task Behavioral Data (Median Response Times $+/-$ S.D.) \\
Median Play Initiation (all trials) & $557.21+/-167.95$ \\
Post Win Median Play Initiation Time & $575.75+/-182.09$ \\
Post Near Miss Median Play Initiation Time & $537.98+/-158.14$ \\
Post Total Miss Median Play Initiation Time & $549.66+/-175.12$ \\
\hline
\end{tabular}

TFA measures from the corresponding ERP time window; each omnibus model was held to Bonferroni-adjusted threshold to account for four comparisons $(\mathrm{p}<.013)$.

Participant Age: Given our wide age range (19-61 years) we assessed the association of age with the electrophysiological measures of reward processing using Bonferroni-corrected Pearson product-moment correlation coefficients.

Relationships between electrophysiological and self-report measures of reward sensitivity were assessed in 2 regression models (TEPS anticipatory and consummatory pleasure), with Bonferroni-correction applied across the two models $(\mathrm{p}<.025)$.

\section{Results}

\subsection{Task behavior}

Response times: There was a significant difference among response times to initiate trials following a win (AAA), near miss (AAB), or total miss $(A B C)$ outcome (condition effect omnibus $p=.003$ ) (Table 1; Fig. 1). Follow-up comparisons among the conditions indicated that participants were faster to initiate trials following a near miss, relative to a win (Bonferroni-corrected, $\mathrm{p}=.003$ ) while response times did not significantly differ among any other pairwise comparisons.

\subsection{ERP Condition effects}

Anticipation (SPN): Prior to R3 onset, a characteristic SPN with a central topography was observed for potentially winning trials (AA; i.e., trials not yet revealed as a win or near miss), relative to total miss (AB) trials (Fig. 2). There was a significant effect of condition, driven by more negative SPN amplitudes at $\mathrm{Cz}$ when comparing $\mathrm{AA}$ to $\mathrm{AB}$ trials $\mathrm{t}$ $(53)=-5.22, \mathrm{p}<.001)$.

Early Outcome Evaluation (RewP): Grand average waveforms (Figs. 2 and 3) show a RewP with expected frontocentral topography in the difference wave of wins (AAA) - near misses (AAB) peaking in the measurement window (228 - 334ms) (Sambrook and Goslin, 2015). There was a significant difference at $\mathrm{FCz}$, with ERP amplitude to wins being larger than to near misses $(\mathrm{t}(53)=8.76, \mathrm{p}<.001)$. In addition, the RewP difference score was larger at Reel 3 (AAA-AAB) than Reel 2 (AA-AB); $(\mathrm{t}(53)=8.40, \mathrm{p}<.001)$.

Late Outcome Evaluation (LPP): Grand average waveforms (Fig. 2) show a sustained positivity with a posterior distribution. Repeated measures ANOVA of the LPP showed a significant main effect of condition at Pz: $\mathrm{F}(2,106)=58.12, \mathrm{p}<.001)$. Bonferroni-corrected follow-up tests across the three outcomes indicated the omnibus condition effect was explained by larger LPP amplitudes to both wins $(\mathrm{p}<.001)$ and near misses ( $\mathrm{p}<.001)$, compared to total misses. Near misses also differed from total misses $(\mathrm{p}=.001)$, with near miss amplitudes falling in-between those of wins and total misses (i.e., wins $>$ near misses $>$ total misses).

See Figs. 2 and 3. 

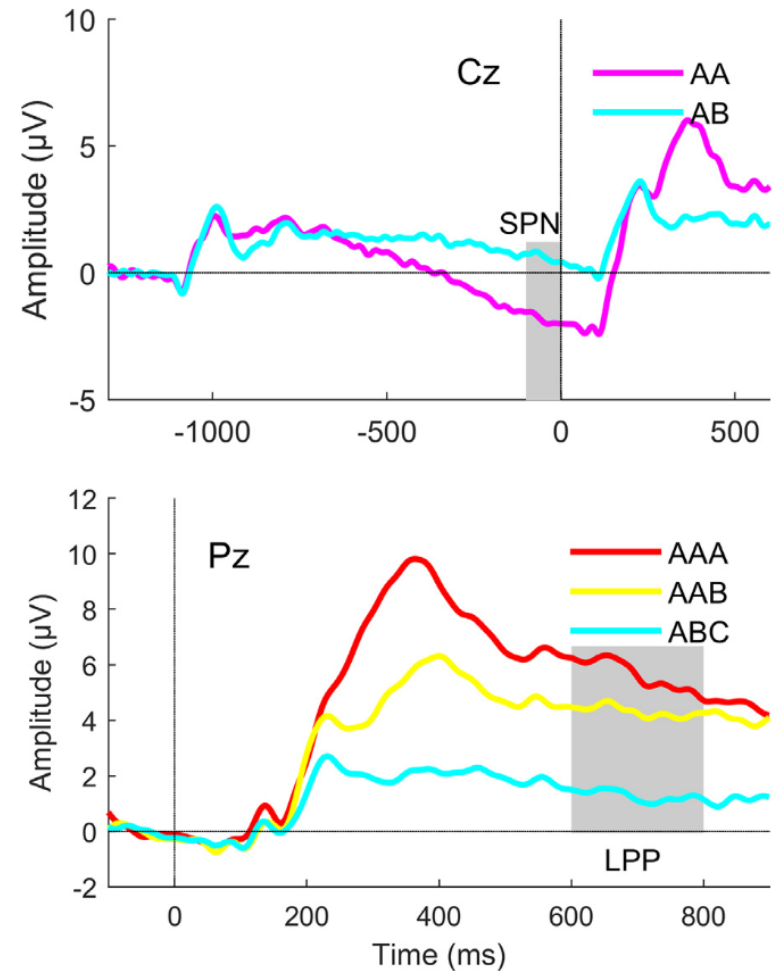

Fig. 2. Top: Stimulus preceding negativity (SPN) grand average waveforms at electrode $\mathrm{Cz}$ depicting Reel 2 time-locked possible win (AA) trials shown in pink and total miss (AB) trials shown in cyan. Bottom: Late positive potential (LPP) grand average waveforms at electrode $\mathrm{Pz}$ for win (AAA) trials shown in red and near miss (AAB) trials shown in yellow, and total miss (ABC) trials shown in cyan. Time $0 \mathrm{~ms}$ corresponds to Reel- 3 outcome. Grey bars depict ERP measurement window; $\mathrm{n}=54$.

\subsection{Time-frequency analysis Condition Effects}

We observed significant condition effects for both theta power and ITC (omnibus $\mathrm{p}<.001$ for theta power and ITC). Bonferroni-corrected follow-up tests across the three outcomes indicated differences were explained by increased power and phase synchrony in response to near misses, relative to both wins (power $\mathrm{t}(53)=4.06, \mathrm{p}<.001$; ITC $\mathrm{t}(53)=5.17, \mathrm{p}<.001$ ) and R2 total misses (power $\mathrm{t}(53)=2.64, \mathrm{p}=.011$; ITC $t(53)=6.38, p<.001)$. Further, theta activity did not distinguish R3 wins from R2 total misses ( $\mathrm{p}>$.07).

Delta power and ITC also showed significant condition effects (omnibus delta power $\mathrm{p}=.002$; delta ITC $\mathrm{p}=.007$ ), with follow-ups indicating greater delta power and ITC to R3-wins relative to R2-total misses (delta power $\mathrm{t}(53)=3.34, \mathrm{p}=.002$; delta ITC $\mathrm{t}(53)=3.08, \mathrm{p}=.003$ ). Delta power was also significantly greater for R3-near misses than R2total misses $(t(53)=2.72, p=.009)$, while wins and near misses did not differ $(\mathrm{p}=.20)$. R3-near misses showed numerically intermediate values of delta ITC, falling between R2-total misses and R3-wins but not significantly differing from either ( $\mathrm{p}>.08$ ). See Fig. 4.

\subsection{Time-frequency measures as predictors of ERPS}

Regression models of ERP component difference scores (SPN, RewP, LPP_wins, LPP_near misses) on theta and delta signals within time windows corresponding to each ERP component were conducted in order to determine the underlying contributions of delta and theta total power and phase synchrony to the time-domain ERP component effects associated with different stages of reward processing. The overall model regressing RewP difference scores on theta power, delta power, theta ITC and delta ITC difference scores (all measured in the RewP time win-
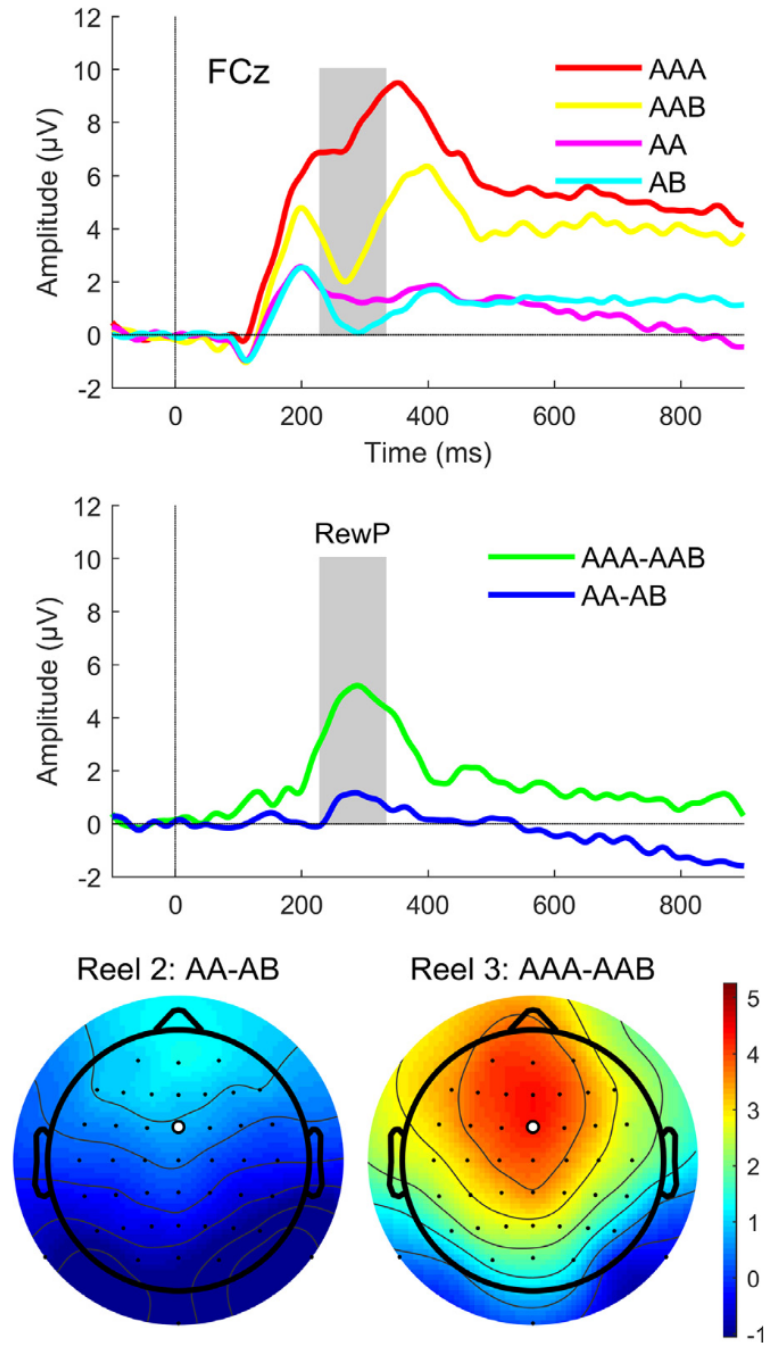

Fig. 3. Effects of Reel Position. Top: Grand average ERP waveforms by condition at electrode FCz. Waveforms for Reel-3 locked (AAA, AAB) and Reel-2 locked $(\mathrm{AA}, \mathrm{AB})$ individual conditions. $0 \mathrm{~ms}$ on the $\mathrm{x}$-axis corresponds to Reel2 (AA, AB) outcome, which spanned from 1494-2000 ms from trial onset and Reel-3 (AAA, AAB) outcome which spanned from 3894-4000 ms from trial onset. Middle: Difference waves for Reel 3-locked AAA-AAB and Reel 2-locked AA-AB comparisons. Grey bar depicts ERP measurement window for Reward Positivity (RewP). Bottom: Topographical maps of condition difference waves at Reel 2 (bottom, left) and Reel 3 (bottom, right).

dow at $\mathrm{FCz}$ ) was significant $\mathrm{F}(4,49)=5.55, \mathrm{p}=.001 ; \mathrm{R}^{2}=.31$. Theta ITC $(\mathrm{t}=-2.63, \mathrm{p}=.01)$ and delta ITC $(\mathrm{t}=2.05, \mathrm{p}=.046)$ difference scores both made significant unique contributions in the model, with delta phase synchrony difference scores showing a positive relationship with the RewP, and theta phase synchrony difference scores showing a negative relationship. Neither power measure difference score was a significant regressor in the model ( $\mathrm{p}>.05)$.

In contrast, the regression models predicting SPN or LPP amplitudes from TFA measures within corresponding time windows were not significant (SPN: $\mathrm{F}(4,49)=1.34, \mathrm{p}=.27 ; \mathrm{R}^{2}=.10$; LPP_wins: $\mathrm{F}(4,49)=0.61$, $\mathrm{p}=.66$; $\mathrm{R}^{2}=.05$; LPP_near misses: $\left.\mathrm{F}(4,49)=1.08, \mathrm{p}=.38 ; \mathrm{R}^{2}=.08\right)$, indicating theta and delta time-frequency measures were not significant predictors of SPN or LPP ERPs.

\subsection{Relationships with age}

Age relationships were examined among the four ERPs (SPN, RewP, LPP_wins, LPP_near misses) and theta and delta power and ITC to wins, 

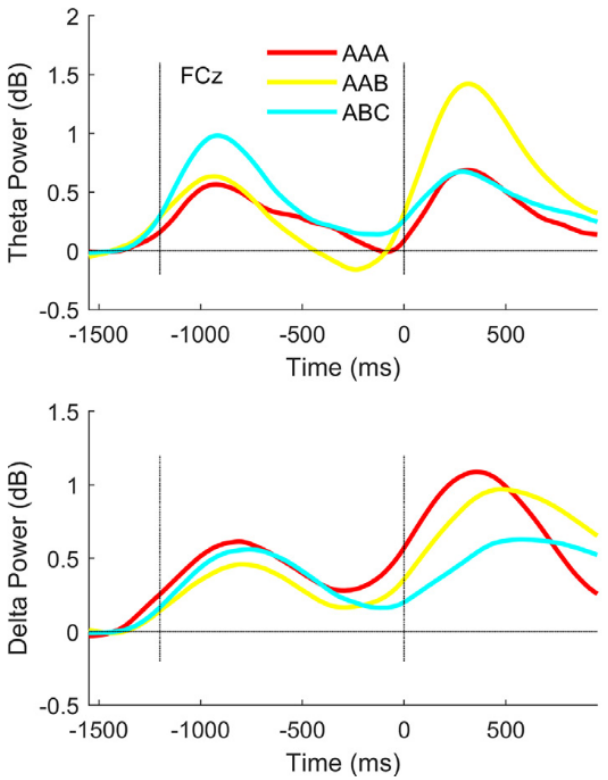
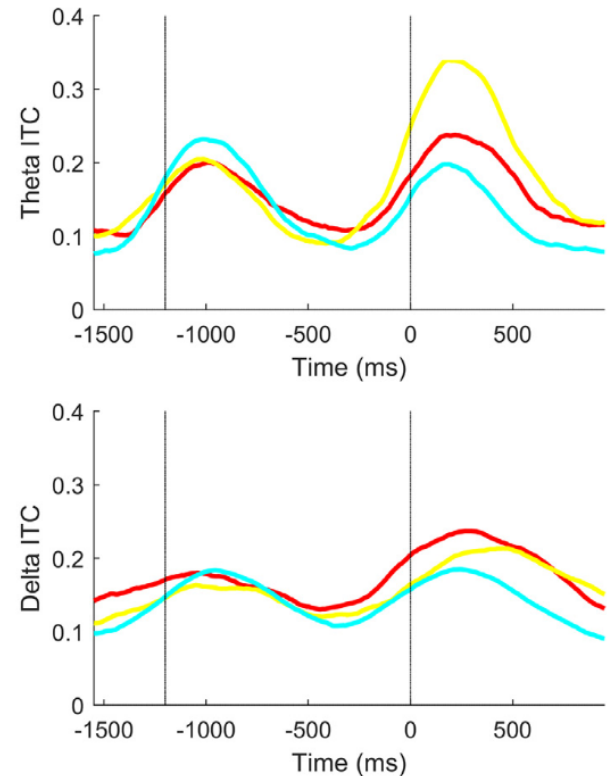

Fig. 4. Condition effects for total power (left) and inter-trial coherence (ITC) (right), for theta (5Hz; Top) and delta $(3 \mathrm{~Hz}$; Bottom) bands. Time 0 ms corresponds to Reel- 3 outcome. ABC trials were downsampled to equate trial numbers across conditions. near misses, and total misses within the RewP time window using a Bonferroni-corrected alpha across these measures $(\mathrm{p}<.006)$. There was a significant negative association between the RewP and age $(r=-0.46$; $\mathrm{p}=.001$ ); no other comparisons surpassed the Bonferroni-adjusted statistical significance threshold $(.03<|\mathrm{r}|<.25 ; 0.07<\mathrm{p}<.82)$.

\subsection{Relationships with behavioral measures of reward sensitivity}

The first model regressed the TEPS-anticipatory onto the ERP anticipatory measure, the SPN, which was not a significant predictor $(\mathrm{t}=$ $0.98, \mathrm{p}=.93$; model $\mathrm{R}^{2}=.01$ ). The second model regressed the TEPSconsummatory measures onto the remaining ERP measures, which represented post-outcome responses (RewP, LPP_wins, LPP _near misses). Here, the LPP_wins was positively associated with variance in TEPS consummatory scores $\left(\mathrm{t}=2.79, \mathrm{p}=.007\right.$; model $\left.\mathrm{R}^{2}=.14\right)$, while betas for the RewP $(\mathrm{t}=-.57, \mathrm{p}=.57)$ and LPP_near misses $(\mathrm{t}=-2.02, \mathrm{p}=.05)$ were not significant.

\section{Discussion}

The goal of this study was to extend prior research on rewardrelated brain functioning by extracting EEG measures of reward anticipation and early and late-stage reward outcome evaluation in the same paradigm, combining ERP and TFA measures in characterizing these sub-components of reward processing, and relating these neurophysiological responses to psychologically distinct phases reflecting wanting (anticipation) vs. liking (consummation). After replicating expected condition effects for ERP and TFA measures of interest, key findings of this study i) extend the literature by demonstrating that delta and theta phase synchrony measures are correlated with RewP, but not SPN or LPP, amplitude, ii) show that the measures under study covary selectively with participant age (RewP) and self-reported sensitivity to consummatory reward processes (LPP_wins), iii) demonstrate that the RewP is larger at R3 than R2, and iv) show that near miss outcomes induce larger and more synchronized frontomedial theta responses than wins and total misses. The slot machine context, which minimizes cognitive and motivational demands, may be particularly relevant for assessing basic reward responsivity in clinical or developmental populations in which within-group variation or case-control differences in motivation and cognition can complicate interpretation of performance-based reward tasks.
Consistent with the literature, we found an SPN precedes R3 on possible win (AA) but not definite loss (AB) trials. This was previously modeled by Donkers in a 3-reel slot paradigm, demonstrating that an SPN precedes, but does not follow, outcome feedback (Donkers et al., 2005, Donkers and Van Boxtel, 2005), and supports the interpretation that, in the context of a reward paradigm, the SPN reflects reward anticipation (Brunia et al., 2011, Brunia and van Boxtel, 2001). The SPN comprises a class of slow negative potentials thought to signify a generalized attentional control response generated during anticipation of impending feedback across varied experimental paradigms (Brunia et al., 2011, Brunia and van Boxtel, 2001). While the SPN is not exclusively generated by incentivized contexts, it is influenced by motivational content of the anticipated feedback (Masaki et al., 2006, Ohgami et al., 2006).

The presence of a RewP with characteristic topography and time signature (Fig. 3) is strongly expected by prior literature across varied reward task paradigms. These are reviewed in (Hauser et al., 2014, Holroyd and Umemoto, 2016, Proudfit, 2015, Walsh and Anderson, 2012) and include studies of slot machine play most relevant to the current study (Donkers et al., 2005, Donkers and Van Boxtel, 2005, Lole et al., 2013, Luo et al., 2011, Qi et al., 2011). Several existing studies used a slot paradigm design with all outcome information being delivered simultaneously (e.g., with trial outcomes defined based on number or proximity of stimuli in the final visual array) (Lole et al., 2013, Luo et al., 2011, Qi et al., 2011). In contrast, our task design utilized sequential reels enabling us to examine the effects of reel position on outcome processing when feedback about loss is revealed sequentially over time, ostensibly allowing anticipation for possible win trials to further develop. Of note, the RewP was the only measure that significantly covaried with age, with younger adults showing larger amplitudes. Many prior studies examining age-related variation in the RewP (or separate win and loss waveforms) have focused on developmental samples, though studies that have extended the age range into middle and older adults observe a pattern similar to ours, with attenuation of the feedback-related waveforms over the course of adulthood (Hammerer et al., 2011, Hill et al., 2018, Nieuwenhuis et al., 2002).

Prior electrophysiological studies have shown that ERP components relevant for reward evaluation such as the RewP are modulated by reward proximity, suggesting that "close" outcomes such as near misses may be processed distinctly from both win and "total miss" events (Lole et al., 2013, Luo et al., 2011, Kreussel et al., 2013, Ulrich and Hewig, 2014). Near misses have also been associated with theta oscillations in reward network regions including the insula and right or- 
bitofrontal cortex (Dymond et al., 2014). However, the R3-locked RewP contains both the reward-related positivity to the win as well as the relative negativity to the near miss. Thus, both may contribute meaningful variance to the RewP as a difference score (Warren and Holroyd, 2012). Because our task design enabled comparing temporally distinct reel outcome effects in the RewP time window, we were able to observe that the RewP is significantly larger at R3 (AAA-AAB) than R2 (AA-AB), despite the equivalent conditional probability of losing at each of these reels and the equivalent economic valuation of a zero payout for losses. Observing a R2 RewP converges with prior work demonstrating the RewP can be elicited by intermediate feedback using a performance-dependent (modified monetary incentive delay) reward task (Threadgill and Gable, 2018). However, though the slot task produces two RewP components, the R3-locked one is larger suggesting that the win on R3 drives the R3 RewP amplitude increase more than the losses (which are present at both reels), fitting with the conceptualization that the RewP predominantly represents win-related signaling (Proudfit, 2015, Holroyd et al., 2008).

We also observed an LPP after the RewP that was affected by trial outcome, being larger to wins (AAA), intermediate (AAB) for near misses, and smallest for total misses ( $A B C$ ), i.e., $A A A>A A B>A B C$ . The LPP has most often been studied in the context of affective processing (e.g., affective picture exposure) and is thought to signify attentional allocation to stimuli with affective content, possibly in the service of facilitating encoding of emotionally relevant stimuli (Hajcak and Olvet, 2008). LPP amplitude covaries with BOLD activations in temporo-parietal and occipital cortices (Sabatinelli et al., 2007) consistent with the component's characteristic posterior scalp topography. However, studies using temporal-spatial PCA have indicated that the large, slow positivity characterized by the LPP can be fractionated into sub-components that have distinct spatial morphologies (e.g., occipital vs. parietal) and may respond selectively to cognitive vs. affective aspects of task stimuli (Foti et al., 2015, Matsuda and Nittono, 2015, Foti et al., 2009). This distinction is supported by Cockburn et al, who focused on the role of feedback quality on RewP amplitude elicited during a time estimation task and found that a posterior slow wave (500$700 \mathrm{~ms}$, post-feedback) became more positive as feedback became more informative (Cockburn and Holroyd, 2018). The authors suggested the slow, late wave they observed could signify higher-order encoding of feedback that may functionally apply the information carried by earlier evaluative components like the RewP to formal learning principles as outcomes are further processed. Indeed, this interpretation is consistent with the idea that the RewP, as an initial reward evaluation response, signals receipt of the reward prediction error into ACC, while later activities may be more tightly coupled to subsequent behavioral adjustment based on the initial processing of feedback (Cavanagh and Shackman, 2015). Though our study had no manipulation of feedback quality or other cognitive manipulation, the LPP to wins significantly related to self-reported consummatory pleasure. In contrast, the SPN was not related to self-reported anticipatory pleasure. LPP response to wins was strongly related to the SPN to possible wins $(r=-.42 ; \mathrm{p}=.002$; data not shown) with larger LPP to wins co-varying with more negative SPN amplitudes. This relationship replicates a prior report using a dissimilar reward task (Pornpattananangkul and Nusslock, 2015), suggesting that SPN and LPP responses, though psychologically and temporally distinct, may share underlying functional features. Similarly, the SPN has also been shown to co-vary with the feedback P3 in a monetary incentive delay task (Novak et al., 2016).

Time-frequency analyses were undertaken on single trial EEG activity to examine the contribution of delta and theta oscillatory bands to observed ERP difference-score condition effects during anticipatory and feedback phases of slot play. First, we found that neither theta nor delta power or phase synchrony were associated with SPN or LPP amplitudes. In contrast, theta and delta phase synchrony measures were both significantly associated with RewP amplitude, with a stronger relationship for theta than delta. Thus, our data suggests that the RewP derives from phase resetting of theta and delta oscillations rather than a change in the magnitude of these oscillations. Our findings support prior conclusions based on analyses of evoked power (Watts et al., 2018, Foti et al., 2015, Nelson et al., 2011) and ITC (Watts et al., 2018) indicating that theta and delta make independent contributions to the time domain RewP. The single trial time-frequency analysis we undertook extends prior work based on time-frequency decomposition of trial averages by examining oscillatory total power and phase synchrony which offers a different account EEG dynamics (Makeig et al., 2004) (e.g., by modeling spectral amplitude perturbations, regardless of stimulus phase). The RewP, or more specifically the FRN, has been theoretically and empirically tied to the more widely studied error-related negativity (ERN), (Falkenstein et al., 1991, Gehring, 1993) a frontomedial negativity elicited by internal error or conflict detection (as opposed to the external feedback that elicits a RewP/FRN); thus the ERN and RewP may share an underlying mechanism that functions as an error detection system (Miltner et al., 1997). Though the RewP is typically defined as a difference score of wins vs. losses, resulting in a relative positivity (reviewed by (Proudfit, 2015)), its amplitude has contributions from both the positivity to wins and the negativity to losses (Holroyd et al., 2008). Our data provide further evidence that there are unique contributions to RewP from theta and delta bands.

Additional time-frequency analyses isolated condition specific effects that would be difficult to disentangle in the temporal domain due to component overlap and large magnitude differences between R2 and R3. Condition-specific phase synchronization and magnitude changes in response to event onsets (ITC and total power) were observed, corresponding with i) increased delta oscillation magnitude and synchronization to wins relative to total misses (with wins and near misses showing equivalent delta signaling) and ii) increased theta oscillation magnitude and synchronization to near misses, relative to both wins and total misses. Two prior studies from the same research group did not show differences in theta power between near misses and wins (Alicart et al., 2015, Alicart et al., 2019), though both were based on a slot task in which near misses occurred with twice the frequency as wins, making it difficult to compare results with our study in which trial numbers were balanced across near miss and win outcomes to promote disentangling brain responses related to outcome processing from probability effects. Our findings support prior studies of reward outcome valence that associate theta with loss-related processing and delta with winning outcomes (Bernat et al., 2015, Bernat et al., 2011, Cohen et al., 2007, MarcoPallares et al., 2008, Watts et al., 2018, Foti et al., 2015, Nelson et al., 2011). Notably, near misses in our data elicited the largest magnitude and phase synchrony of the frontomedial theta response, with significantly more theta power and synchrony than wins but also significantly more than for total misses (i.e., losses revealed at R2). Furthermore, subsequent play behavior was influenced by outcome on the preceding trial as play initiation times following a near miss were significantly faster than initiation times subsequent to a win. This effect could be interpreted as a post-reinforcement pause following winning trials that has been described in the literature (Delfabbro and Winefield, 1999, Dixon et al., 2013). This effect is also consistent with the possibility of a frustration effect (Amsel, 1958) being induced by near misses that invigorates future play, as others have suggested in the context of gambling near misses (Reid, 1996, Dixon et al., 2013). That is, because near misses come closer to wins than other types of losses, near miss effects may elicit a frustrative non-reward response, in which failure to obtain a desired goal invigorates behavior (Amsel, 1958). That our data demonstrate that R3 "near miss" loss elicits more theta signaling than total misses despite the equivalent economic valuation and conditional probability of both loss outcomes, highlights the sensitivity of theta signals to reward proximity, and suggests a possible electrophysiological mechanism underlying frustrative non-reward.

One important study limitation is that theta and delta bands were chosen a priori based on prior evidence of involvement in reward-related processing (Bernat et al., 2015, Bernat et al., 2011, Cavanagh et al., 
2010, Cohen et al., 2007, Cavanagh, 2015); and so we cannot rule out the possibility that frequency bands that we did not examine explain variance in the SPN or LPP, or additional variance in the RewP. Future research in the frequency domain focusing comprehensively on all bands and time points, including cross-frequency coupling is indicated. In addition, combined EEG-fMRI analyses will be useful to characterize neuroanatomy relevant to these EEG measures. Further, while individual differences were detected with ERP measures and participant age (RewP) and reward sensitivity (LPP), attenuation of the true correlation magnitude of these relationships could have resulted from the restricted range of the HC sample, which should be examined in future studies assessing wider ranges of age and reward functioning. Taken together, these results highlight the differential contributions of ERP and TFA measures to psychologically distinct aspects of reward processes involved in anticipating and evaluating rewards, and add to the literature characterizing reward processing features during slot play, including the near miss response.

\section{Disclosure}

The authors declare no competing interests. DHM is a consultant to Boehringer Ingelheim Pharmaceuticals, Syndisi, Cadent Therapeutics, and Recognify.

\section{Data availability}

Data and task paradigm available from the corresponding author upon reasonable request. Our informed consent procedure specified that de-identified data may be shared with collaborators and our institutional policy requires collaborators to enter a formal data sharing agreement. The following software used in our analyses is publicly available: Brain Vision Analyzer 2.0 (Brain Products, www.brainproducts.com), MATLAB R2019a (MathWorks; https://www.mathworks.com), EEGLAB (Swartz Center for Computational Neuroscience, UCSD; https://sccn.ucsd.edu/eeglab).

\section{Acknowledgements}

VA: CX001028 and CX001980 to (SLF). Drs. Fryer, Ford, and Mathalon are employees of the U.S. Government. The content is solely the responsibility of the authors and does not necessarily represent the views of the Department of Veterans Affairs.

\section{Supplementary materials}

Supplementary material associated with this article can be found, in the online version, at doi:10.1016/j.neuroimage.2021.117874.

\section{References}

Alicart, H., Cucurell, D., Mas-Herrero, E., Marco-Pallares, J., 2015. Human oscillatory activity in near-miss events. Soc. Cogn. Affect. Neurosci. 10, 1405-1412.

Alicart, H., Mas-Herrero, E., Rifa-Ros, X., Cucurell, D., Marco-Pallares, J., 2019. Brain oscillatory activity of skill and chance gamblers during a slot machine game. Cogn. Affect. Behav. Neurosci. 19, 1509-1520.

Amsel, A., 1958. The role of frustrative nonreward in noncontinuous reward situations. Psychol. Bull. 55.

Barch, D.M., Pagliaccio, D., Luking, K., 2016. Mechanisms underlying motivational deficits in psychopathology: similarities and differences in depression and schizophrenia. Curr. Top. Behav. Neurosci. 27, 411-449.

Barton, K. R. et al. The effect of losses disguised as wins and near misses in electronic gaming machines: a systematic review. J. Gambl. Stud. 33, 1241-1260, (2017).

Becker, M.P., Nitsch, A.M., Miltner, W.H., Straube, T., 2014. A single-trial estimation of the feedback-related negativity and its relation to BOLD responses in a time-estimation task. J. Neurosci. 34, 3005-3012.

Bernat, E.M., Nelson, L.D., Steele, V.R., Gehring, W.J., Patrick, C.J., 2011. Externalizing psychopathology and gain-loss feedback in a simulated gambling task: dissociable components of brain response revealed by time-frequency analysis. J. Abnorm. Psychol. 120, 352-364.

Bernat, E.M., Nelson, L.D., Baskin-Sommers, A.R., 2015. Time-frequency theta and delta measures index separable components of feedback processing in a gambling task. Psychophysiology 52, 626-637.
Berridge, K.C., Kringelbach, M.L., 2015. Pleasure systems in the brain. Neuron 86, 646-664.

Berridge, K.C., Robinson, T.E., 2003. Parsing reward. Trends Neurosci. 26, 507-513.

Brunia, C.H., van Boxtel, G.J., 2001. Wait and see. Int. J. Psychophysiol. 43, 59-75.

Brunia, C.H., Hackley, S.A., van Boxtel, G.J., Kotani, Y., Ohgami, Y., 2011. Waiting to perceive: reward or punishment? Clin. Neurophysiol. 122, 858-868.

Carlson, J.M., Foti, D., Mujica-Parodi, L.R., Harmon-Jones, E., Hajcak, G., 2011. Ventral striatal and medial prefrontal BOLD activation is correlated with reward-related electrocortical activity: a combined ERP and fMRI study. Neuroimage 57, 1608-1616.

Cavanagh, J.F., Frank, M.J., 2014. Frontal theta as a mechanism for cognitive control. Trends Cogn. Sci. 18, 414-421.

Cavanagh, J.F., Shackman, A.J., 2015. Frontal midline theta reflects anxiety and cognitive control: meta-analytic evidence. J. Physiol. Paris 109, 3-15.

Cavanagh, J.F., Frank, M.J., Klein, T.J., Allen, J.J., 2010. Frontal theta links prediction errors to behavioral adaptation in reinforcement learning. Neuroimage 49, 3198-3209.

Cavanagh, J.F., 2015. Cortical delta activity reflects reward prediction error and related behavioral adjustments, but at different times. Neuroimage 110, 205-216.

Christie, G.J., Tata, M.S., 2009. Right frontal cortex generates reward-related theta-band oscillatory activity. Neuroimage $48,415-422$

Clark, L., Lawrence, A.J., Astley-Jones, F., Gray, N., 2009. Gambling near-misses enhance motivation to gamble and recruit win-related brain circuitry. Neuron 61, 481-490.

Cockburn, J., Holroyd, C.B., 2018. Feedback information and the reward positivity. Int. J. Psychophysiol. 132, 243-251.

Cohen, M.X., Elger, C.E., Ranganath, C., 2007. Reward expectation modulates feedback-related negativity and EEG spectra. Neuroimage 35, 968-978.

Delfabbro, P.H., Winefield, A.H., 1999. Poker-machine gambling: an analysis of within session characteristics. Br. J. Psychol. 90, 425-439.

Dixon, M.R., MacLaren, V., Jarick, M., Fugelsang, J.A., Harrigan, K.A., 2013. The frustrting effects of just missing the jackpot: Slot machine near-misses trigger large skin conductance responses, but no post-reinforcement pauses. J. Gambl. Stud. 29, 661-674.

Donkers, F.C., Van Boxtel, G.J., 2005. Mediofrontal negativities to averted gains and losses in the slot-machine task: a further investigation. J. Psychophysiol. 19, 256-262.

Donkers, F.C., Nieuwenhuis, S., van Boxtel, G.J., 2005. Mediofrontal negativities in the absence of responding. Brain Res. Cogn. Brain Res. 25, 777-787.

Dymond, S., et al., 2014. Almost winning: induced MEG theta power in insula and orbitofrontal cortex increases during gambling near-misses and is associated with BOLD signal and gambling severity. Neuroimage 91, 210-219.

Falkenstein, M., Hohnsbein, J., Hoormann, J., Blanke, L., 1991. Effects of crossmodal divided attention on late ERP components. II. Error processing in choice reaction tasks. Electroencephalogr Clin. Neurophysiol. 78, 447-455.

First, M., Spitzer, R., Gibbon, M., Williams, J., 2002. Structured Clinical Interview for DSM-IV-TR Axis I Disorders, Research Version, Patient Edition. (SCID-I/P). New York State Psychiatric Institute.

Foti, D., Hajcak, G., Dien, J., 2009. Differentiating neural responses to emotional pictures: evidence from temporal-spatial PCA. Psychophysiology 46, 521-530.

Foti, D., Weinberg, A., Bernat, E.M., Proudfit, G.H., 2015. Anterior cingulate activity to monetary loss and basal ganglia activity to monetary gain uniquely contribute to the feedback negativity. Clin. Neurophysiol. 126, 1338-1347.

Gard, D.E., Gard, M.G., Kring, A.M., John, O.P., 2006. Anticipatory and consummatory components of the experience of pleasure: a scale development study. J. Res. Personal. 40, 1086-1102.

Gehring, W.J., Willoughby, A.R., 2002. The medial frontal cortex and the rapid processing of monetary gains and losses. Science 295, 2279-2282.

Gehring, W.J., 1993. A neural system for error detection and compensation. Psychol. Sci. $4,385-390$.

Glazer, J.E., Kelley, N.J., Pornpattananangkul, N., Mittal, V.A., Nusslock, R., 2018. Beyond the FRN: Broadening the time-course of EEG and ERP components implicated in reward processing. Int. J. Psychophysiol. 132, 184-202.

Griffiths, M., 1993. Fruit machine gambling: The importance of structural characteristics. J. Gambl. Stud. 9.

Gruber, M.J., Watrous, A.J., Ekstrom, A.D., Ranganath, C., Otten, L.J., 2013. Expected reward modulates encoding-related theta activity before an event. Neuroimage 64, 68-74.

Habib, R., Dixon, M.R., 2010. Neurobehavioral evidence for the "Near-Miss" effect in pathological gamblers. J. Exp. Anal. Behav. 93, 313-328.

Hajcak, G., Olvet, D.M., 2008. The persistence of attention to emotion: brain potentials during and after picture presentation. Emotion 8, 250-255.

Hammerer, D., Li, S.C., Muller, V., Lindenberger, U., 2011. Life span differences in electrophysiological correlates of monitoring gains and losses during probabilistic reinforcement learning. J. Cogn. Neurosci. 23, 579-592.

Hauser, T.U., et al., 2014. The feedback-related negativity (FRN) revisited: new insights into the localization, meaning and network organization. Neuroimage 84, 159-168.

Hill, K.E., Ait Oumeziane, B., Novak, K.D., Rollock, D., Foti, D., 2018. Variation in rewardand error-related neural measures attributable to age, gender, race, and ethnicity. Int. J. Psychophysiol. 132, 353-364.

Holroyd, C.B., Coles, M.G.H, 2002. The neural basis of human error processing: reinforcement learning, dopamine, and the error-related negativity. Psychol. Rev. 109, 679-709.

Holroyd, C.B., Umemoto, A., 2016. The research domain criteria framework: the case for anterior cingulate cortex. Neurosci. Biobehav. Rev. 71, 418-443.

Holroyd, C.B., Pakzad-Vaezi, K.L., Krigolson, O.E., 2008. The feedback correct-related positivity: sensitivity of the event-related brain potential to unexpected positive feedback. Psychophysiology 45, 688-697.

Knutson, B., Westdorp, A., Kaiser, E., Hommer, D., 2000. FMRI visualization of brain activity during a monetary incentive delay task. Neuroimage 12, 20-27. 
Kort, N.S., et al., 2017. Role of N-Methyl-D-aspartate receptors in action-based predictive coding deficits in schizophrenia. Biol. Psychiatry 81, 514-524.

Kreussel, L., et al., 2013. How bad was it? Differences in the time course of sensitivity to the magnitude of loss in problem gamblers and controls. Behav. Brain Res. 247, 140-145.

Leonowicz, Z., Karvanen, J., Shishkin, S.L., 2005. Trimmed estimators for robust averaging of event-related potentials. J. Neurosci. Methods 142, 17-26.

Lole, L., Gonsalvez, C.J., Barry, R.J., De Blasio, F.M., 2013. Can event-related potentials serve as neural markers for wins, losses, and near-wins in a gambling task? A principal components analysis. Int. J. Psychophysiol. 89, 390-398.

Lopez-Calderon, J., Luck, S.J., 2014. ERPLAB: an open-source toolbox for the analysis of event-related potentials. Front Hum Neurosci 8, 213.

Luo, Q., Wang, Y., Qu, C., 2011. The near-miss effect in slot-machine gambling: modulation of feedback-related negativity by subjective value. Neuroreport 22, 989-993.

Makeig, S., Debener, S., Onton, J., Delorme, A., 2004. Mining event-related brain dynamics. Trends Cogn. Sci. 8, 204-210.

Marco-Pallares, J., et al., 2008. Human oscillatory activity associated to reward processing in a gambling task. Neuropsychologia 46, 241-248.

Masaki, H., Takeuchi, S., Gehring, W.J., Takasawa, N., Yamazaki, K., 2006. Affective-motivational influences on feedback-related ERPs in a gambling task. Brain Res. 1105, 110-121.

Mathalon, D.H., et al., 2018. Deficient auditory predictive coding during vocalization in the psychosis risk syndrome and in early illness schizophrenia: the final expanded sample. Psychol. Med. 1-8.

Matsuda, I., Nittono, H., 2015. Motivational significance and cognitive effort elicit different late positive potentials. Clin. Neurophysiol. 126, 304-313.

Miltner, W.H., Braun, C.H., Coles, M.G., 1997. Event-related brain potentials following incorrect feedback in a time-estimation task: evidence for a "generic" neural system for error detection. J. Cogn. Neurosci. 9, 788-798.

Mognon, A., Jovicich, J., Bruzzone, L., Buiatti, M., 2011. ADJUST: An automatic EEG artifact detector based on the joint use of spatial and temporal features. Psychophysiology 48, 229-240.

Nelson, L.D., Patrick, C.J., Collins, P., Lang, A.R., Bernat, E.M., 2011. Alcohol impairs brain reactivity to explicit loss feedback. Psychopharmacology (Berl) 218, 419-428.

Nelson, B.D., et al., 2018. Time-frequency reward-related delta prospectively predicts the development of adolescent-onset depression. Biol. Psychiatry Cogn. Neurosci. Neuroimaging 3, 41-49.

Nieuwenhuis, S., et al., 2002. A computational account of altered error processing in older age: dopamine and the error-related negativity. Cogn. Affect. Behav. Neurosci. 2, 19-36.

Nolan, H., Whelan, R., Reilly, R.B., 2010. FASTER: fully automated statistical thresholding for EEG artifact rejection. J. Neurosci. Methods 192, 152-162.

Novak, K.D., Foti, D., 2015. Teasing apart the anticipatory and consummatory processing of monetary incentives: An event-related potential study of reward dynamics. Psychophysiology $52,1470-1482$.
Novak, B.K., Novak, K.D., Lynam, D.R., Foti, D., 2016. Individual differences in the time course of reward processing: stage-specific links with depression and impulsivity. Biol. Psychol. 119, 79-90.

Nusslock, R., Alloy, L.B., 2017. Reward processing and mood-related symptoms: An RDoC and translational neuroscience perspective. J. Affect. Disord. 216, 3-16.

Ohgami, Y., et al., 2006. Effects of monetary reward and punishment on stimulus-preceding negativity. Psychophysiology 43, 227-236.

Oostenveld, R., Fries, P., Maris, E., Schoffelen, J.M.FieldTrip, 2011. Open source software for advanced analysis of MEG, EEG, and invasive electrophysiological data. Comput. Intell. Neurosci. 2011, 156869.

Parke, J., Griffiths, M., 2006. The psychology of the fruit machine: the role of structural characteristics (Revisited). International J. Mental Health and Addiction 4, 151-179.

Pornpattananangkul, N., Nusslock, R., 2015. Motivated to win: Relationship between anticipatory and outcome reward-related neural activity. Brain Cogn. 100, 21-40.

Proudfit, G.H., 2015. The reward positivity: from basic research on reward to a biomarker for depression. Psychophysiology 52, 449-459.

Qi, S., Ding, C., Song, Y., Yang, D., 2011. Neural correlates of near-misses effect in gambling. Neurosci. Lett. 493, 80-85.

Reid, R.L., 1996. The psychology of the near miss. J. Gambl. Behav. 2, 32-39.

Sabatinelli, D., Lang, P.J., Keil, A., Bradley, M.M., 2007. Emotional perception: correlation of functional MRI and event-related potentials. Cereb. Cortex 17, 1085-1091.

Sambrook, T.D., Goslin, J., 2015. A neural reward prediction error revealed by a metaanalysis of ERPs using great grand averages. Psychol. Bull. 141, 213-235.

Tallon-Baudry, C., Bertrand, O., Delpuech, C., Permier, J., 1997. Oscillatory gamma-band $(30-70 \mathrm{~Hz})$ activity induced by a visual search task in humans. J. Neurosci. 17, 722-734.

Threadgill, A.H., Gable, P.A., 2018. The sweetness of successful goal pursuit: Approachmotivated pregoal states enhance the reward positivity during goal pursuit. Int. J. Psychophysiol. 132, 277-286.

Ulrich, N., Hewig, J., 2014. A miss is as good as a mile? Processing of near and full outcomes in a gambling paradigm. Psychophysiology 51, 819-823.

Walsh, M.M., Anderson, J.R., 2012. Learning from experience: event-related potential correlates of reward processing, neural adaptation, and behavioral choice. Neurosci. Biobehav. Rev. 36, 1870-1884.

Warren, C.M., Holroyd, C.B., 2012. The impact of deliberative strategy dissociates ERP components related to conflict processing vs. reinforcement learning. Front. Neurosci. $6,43$.

Watts, A.T.M., Tootell, A.V., Fix, S.T., Aviyente, S., Bernat, E.M, 2018. Utilizing time-frequency amplitude and phase synchrony measure to assess feedback processing in a gambling task. Int. J. Psychophysiol. 132, 203-212.

Whitton, A.E., Treadway, M.T., Pizzagalli, D.A., 2015. Reward processing dysfunction in major depression, bipolar disorder and schizophrenia. Curr. Opin. Psychiatry 28, 7-12. 